\title{
Lattice Boltzmann Analysis of the Flow Reduction Mechanism in Stented Cerebral Aneurysms for the Endovascular Treatment
}

\author{
Miki Hirabayashi ${ }^{1}$, Makoto Ohta ${ }^{2}$, Daniel A. Rüfenacht ${ }^{2}$, and Bastien \\ Chopard $^{1}$ \\ 1 University of Geneva, Computer Science Department, 24 rue General-Dufour \\ CH-1211 Geneva 4, Switzerland \\ Miki.Hirabayahsi@cui .unige.ch, \\ 2 Hospital University of Geneva (HUG), Neuroradiology, 24 rue Micheli-du-Crest \\ CH-1211 Geneva 14, Switzerland
}

\begin{abstract}
Two-dimensional numerical analysis of local hemodynamics on flow-reduction mechanism by stent implantation in cerebral aneurysms is presented to understand these interesting hydrodynamic phenomena and improve this promising minimally invasive treatment. Recently in the cerebral aneurysm treatment, this new endovascular occlusion technique using a porous tubular shaped stent or coils sometimes replaces invasive open surgeries. It is thought that the flow reduction by the stent implantation prevents the aneurysm rupture, however its mechanism is not well understood. We reveal the fundamental flow reduction mechanism by the stent implantation in dependence of the aneurysm size using the lattice Boltzmann approach.
\end{abstract}

\section{Introduction}

Physiological-fluid problems have attracted a strong interest, not only because of their practical importance, but also because of the intriguing physiological phenomena [1-4]. Most of such problems do not have a clear solution, therefore, numerical simulation can provide us with a useful tool for a deeper understanding. Here we focus on local hemodynamics in stented saccular cerebral aneurysms. The stent implantation is the promising minimally invasive endovascular technique and can sometimes be utilized successfully for inoperable lesions. The stent (Fig. 1 (a)), which is a flexible cylindrical mesh tube made of stainless steel or alloys, is implanted, sometimes with coils, in the cerebral arteries (see Fig.1 (b)-(c)) in order to prevent further rupture of aneurysms leading to hemorrhage. It is thought that stent-induced flow stagnation promotes the formation of a stable thrombus in the pouch and leads to permanent occlusion of aneurysm [5]. The stent implantation treatments in cerebral aneurysms are the new treatment and therefore their complex hemodynamics mechanisms involved promotion of thrombosis are not yet well understood. The first experimental and clinical studies of this occlusion technique using stents in carotid aneurysms 
have been done by Marks et al. and Wakhloo et al. in 1994 [6, 7], and several experimental and numerical studies on stented flows have been reported [5, 8-10]. They emphasize the existence of large coherent vortex structures within lateral aneurysm models, however they do not discuss the flow reduction mechanism (i.e. the total reduction of the flux through the stent) well.

Here we mainly consider two types of saccular aneurysms with different neck size in order to investigate the influence of the neck size on the velocity reduction based on the experimental observation [11]. The Reynolds number range in parent vessels $2-4 \mathrm{~mm}$ in diameter is between 100 and 300 and within the aneurysms is less or about $30[8,11]$. For simplicity, flow is considered to be incompressible, homogeneous and nonpulsatile. Body forces such as gravity, boundary forces such as pressure in the brain and the wall elasticity are neglected. The fluid is assumed to be Newtonian. As we mention in Sect. 2, we think these parameters make only minor differences for the purpose of this study [5,8]. Using the two-dimensional lattice Boltzmann approach, we reveal the fundamental flow reduction mechanism by stent implantation in dependence of the aneurysm size. Understanding these flow reduction mechanisms is very important for improvement of the curative effect.

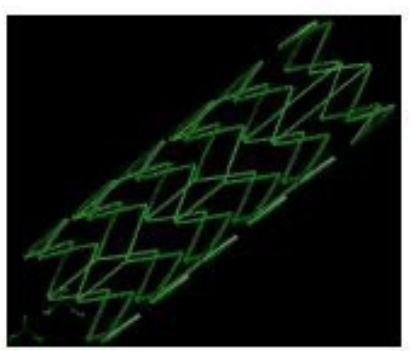

(a)

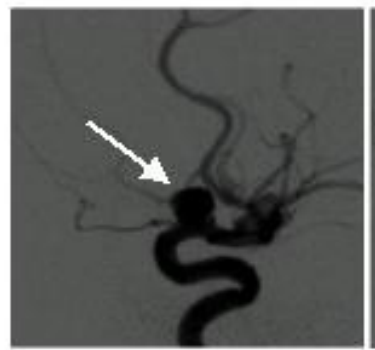

(b)

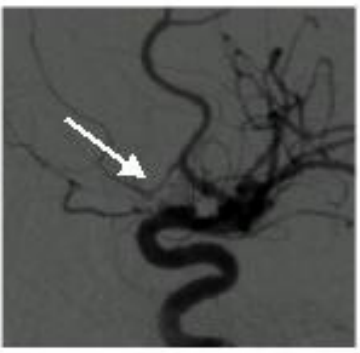

(c)

Fig. 1. A porous tubular shaped stent in (a). Angiogram of the carotid artery before the treatment in (b). A saccular aneurysm is noted (arrow). Angiogram after occlusion using a stent and coils in (c). No flow is recognized in the aneurysm (arrow).

\section{Model Description}

Even without stent the velocity magnitude is low $(1 \mathrm{~cm} / \mathrm{s})$ and the Reynolds number within the aneurysm dome is less or about $30[8,11]$ which characterizes a slow visco-dominated Stokes flow. However, we consider here the inertial term of Navier-Stokes equation in order to describe the vortex structure in aneurysms. We adopt the lattice Boltzmann method $[12,13]$ to solve the following incompressible Navier-Stokes equations in order to investigate fluid dynamics of blood 
1046 M. Hirabayashi et al.

flows.

$$
\rho\left(\partial_{t} \mathbf{u}+\mathbf{u} \cdot \nabla \mathbf{u}\right)=\mu \nabla^{2} \mathbf{u}-\nabla p
$$

The conservation of mass applied to an incompressible flow:

$$
\nabla \cdot \mathbf{u}=0
$$

Here $\mathbf{u}$ is the macroscopic velocity of the fluid, $p$ the local pressure, $\rho$ the density, and $\mu$ the viscosity coefficient. We assume here that the viscosity is constant. The reason is that the non-Newtonian behavior of blood affects the viscosity significantly only at low shear rates, because the aggregation of the red cells causes the increase of the viscosity at the low shear rates, and the influence of shear rates on the viscosity is thought to be relatively small for vessel diameters larger than $0.5 \mathrm{~mm}$, $[8,14]$. Moreover, when we focus on the basic flow dynamics, the difference between non-Newtonian and Newtonian behavior is minor [8,15]. On the other hand, when we consider the promotion of thrombosis in the aneurysm dome where the shear rate is very small, we must consider non-Newtonian behavior.

The lattice Boltzmann algorithm solves the following discretized equation, which recovers the Navier-Stokes equation [12,13]:

$$
n_{i}\left(\mathbf{x}+\mathbf{c}_{i} \delta t, t+\delta t\right)=n_{i}(\mathbf{x}, t)+\Delta_{i}[n(\mathbf{x}, t)]
$$

where the discretized distribution functions $n_{i}(\mathbf{x}, t)$ are considered to represent the positive real-valued number of imaginary particles at a site $\mathbf{x}$ and time $t$ with velocity $\mathbf{c}_{i}$ along the $i$ th direction of the lattice. Here we define the $\delta x / \delta t=\left|\mathbf{c}_{i}\right|$, where $\delta t$ and $\delta x$ are time and space increments, respectively. The term $\Delta_{i}$ is the collision operator, which is usually replaced by its linear expansion around the equilibrium function $n_{i}^{(e q)}(\mathbf{x}, t)[16]$ and simplified using the single-relaxation time as follows $[17,18]$.

$$
\Delta_{i}[n(\mathbf{x}, t)]=-\frac{n_{i}(\mathbf{x}, t)-n_{i}^{(e q)}(\mathbf{x}, t)}{\tau} .
$$

This is called the lattice BGK collision term [19], which represents the relaxation to the equilibrium state. Then we can describe the general form of the equilibrium distribution on the basis of a low Mach number approximation to the expansion of the Maxwellian distribution [20]. We use a two-dimensional model in order to simplify analysis of the flow reduction and to extract the fundamental property of stents. In order to recover the correct Navier-Stokes equations we consider the two-dimensional square lattice with nine velocities, called D2Q9: $\mathbf{c}_{i}=\left(\cos (\pi / 2(i-1)), \sin (\pi / 2(i-1))\right.$ for $i=1,3,5,7, \mathbf{c}_{i}=$ $\sqrt{2}(\cos (\pi / 2(i-1)+\pi / 4), \sin (\pi / 2(i-1)+\pi / 4))$ for $i=2,4,6,8, \mathbf{c}_{0}=0$ corresponds to zero-speed velocity. We can obtained the equilibrium function analytically [13]:

$$
n_{i}^{(e q)}=\rho w_{i}\left[1+3 \mathbf{c}_{i} \cdot \mathbf{u}+\frac{9}{2}\left(\mathbf{c}_{i} \cdot \mathbf{u}\right)^{2}-\frac{3}{2} u^{2}\right],
$$


with $w_{o}=4 / 9, w_{1}=w_{3}=w_{5}=w_{7}=1 / 9$, and $w_{2}=w_{4}=w_{6}=w_{8}=1 / 36$. The constant sound speed $c_{s}=1 / \sqrt{3}$ and the kinetic viscosity $\nu=\mu / \rho=$ $c_{s}^{2} \delta t\left(\tau-\frac{1}{2}\right)=(2 \tau-1) / 6$ [13]. This method solves the equation based on the microscopic dynamics and therefore it is appropriate to solve the macroscopic hydrodynamic phenomena based on the microscopic physics.

Here we use the following parameters for the calculation. The Reynolds number is defined as $R e=\frac{U L}{\nu}$, where $U$ is the characteristic velocity and $L$ is the characteristic length. The velocity at the center of the channel is chosen for $U$ and the channel width is $L$. In order to obtain the appropriate Reynolds number $R e=300$ in the parent vessel flow, we use the Mach number $M<0.2$ instead of the typical Mach number $M=3.0 \times 10^{-5}$ in the parent vessel $2-4 \mathrm{~mm}$ in diameter. The relaxation time $\tau=0.58$ is chosen to produce a constant kinetic viscosity $\nu=(2 \tau-1) / 6=0.026$. The average density of the system is $\rho=1.0$. The simulation size is ranged from $800 \times 288$ cells to $800 \times 188$ cells and the channel width is 80 cells (see Fig. 2 and Table 1 ). Since the velocity profile at the inlet/outlet is unknown it is necessary to specify the pressure gradient across the inlet/outlet instead. The pressure is part of the stress tensor, interpreted as a surface force acting normal to the inflow/outflow surfaces, therefore the inflow/outflow boundary condition is specified as values of the normal component of the stress tensor. We use the constant pressure gradient $\frac{d p}{d x} \cong 4.17 \times 10^{-6}$ rather than a pulsatile flow in order to simplify the study of flow reduction by stents. We expect to obtain the velocity in the center of channel $U=-\frac{L^{2}}{8 \mu} \frac{d p}{d x}$ for the two-dimensional Poiseuille flow [21]. For the inlet and outlet, we use periodic boundaries in combination with velocity and pressure boundaries [22]. For the walls, a number of boundary conditions have been proposed [13,23, 24]. We use the bounce-back on the links, in which particles coming to the walls simply return back to the fluid in the direction where they came from [21].

The geometric dimensions of the aneurysm and the stent (see Table 1) are chosen in the range of experimental data $[7,11]$ and of the clinical observations. The aneurysm diameter varies from $5 \mathrm{~mm}$ to $10 \mathrm{~mm}$ and the aneurysm orifice diameter is between $5 \mathrm{~mm}$ and $10 \mathrm{~mm}$. The parent vessel is assumed to be a straight tube of $4 \mathrm{~mm}$ in diameter. The stent diameter is $4 \mathrm{~mm}$ and the length ranges from $25 \mathrm{~mm}$ to $30 \mathrm{~mm}$ in the expanded state. The stents are composed of weave loops made of stainless steal or alloy filament $0.1 \mathrm{~mm}$ in caliber. We use the same no-slip boundary condition on the stent surfaces as that on the walls (see Fig. 2 and Table 1).

In addition the following simplifications are implemented in the models. Body forces (gravitational forces) and boundary forces (pressure in the brain)are neglected. The experimental study using aneurysms overlying and underlying the parent vessel reported that gravity plays a negligible role in flow patterns[5]. We assume the walls to be nonelastic. It was reported that the basic vortex patterns are similar in both rigid and distensible saccular aneurysms [25]. During the diastolic phase only minor differences could be observed. And cerebral arteries are considered to be less distensible than large systemic arteries [26, 27]. Therefore we think that the elasticity of walls does not make large difference in the basic 
flow pattern with stents. On the other hand we think that elasticity should be consider when we study the rupture mechanism based on the elasticity or the influence of the pressure on the wall.

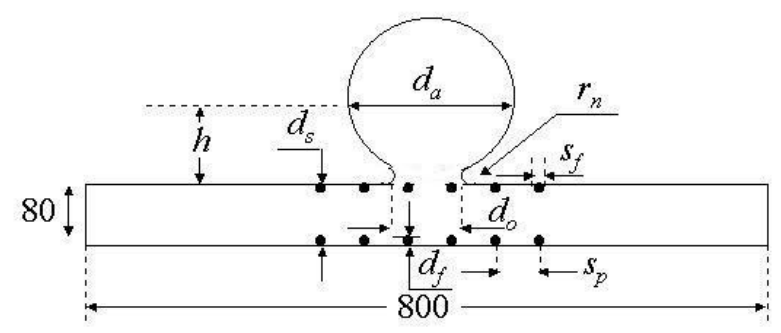

Fig. 2. Geometric parameters of the aneurysm model with a stent.

Table 1. Aneurysm parameters and stent parameters for the numerical experiments. $d_{a}$ denotes the aneurysm diameter, $d_{o}$ is the aneurysm orifice diameter, $d_{s}$ is the stent diameter, $d_{f}$ is the filament diameter, $s_{p}$ is the pore size, $s_{f}$ is the pore interval, and $\varepsilon$ is the geometrical porosity (metal free area/total unit area) of the stent (see Fig. 2). We use other stents with the different pore size in Fig. 5

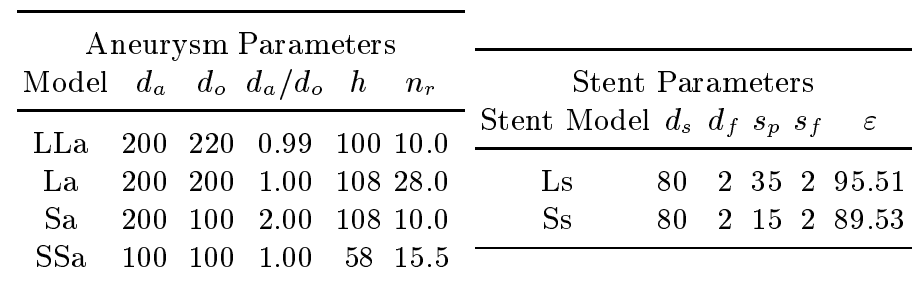

\section{$3 \quad$ Numerical Results}

In this section we present the two-dimensional numerical simulation result performed using the lattice Boltzmann algorithm. We study the hemodynamic effects of stent implantation on several aneurysms and reveal its flow reduction mechanism and critical properties in dependence of the aneurysm size.

Figures 3 (a)-(f) show the variation of the flow patterns in stented aneurysms with the large (model LLa) orifice and the small orifice (model Sa) using the 
stream-line plot. In Figs. 3 (a) and (d), the vortex in the nonstented aneurysm is driven directly by the flow in the parent vessel. In Figs. 3 (b)-(c) and (e)(f), the vortex is not driven by the orifice flow directly because the velocity at the orifice does not have the enough magnitude to drive it (see Fig. 4 and Table 2). In Figs. 3 (g)-(l), we show the stream-line plots of the stent effect in the aneurysm models La and SSa, which have the same proportion of the aneurysm diameter $\left(d_{a}\right)$ to the aneurysmal orifice diameter $\left(d_{o}\right)$. Two aneurysms have the similar flow patterns. Figures 3 (f) and (l) show that the existence of the vortex depends on $d_{a} / d_{o}$. Figures 3 (f) and (l) also show that the vortex in the stented aneurysm appears when the aneurysm diameter is large. It can be thought that this stented vortex is driven by the existence of the pressure difference in the aneurysm where the percolation dominant velocity, which is the velocity at the no-vortex part in the aneurysm, is small in comparison with the magnitude of the pressure difference (see Table 2). In Table 2 we use the following definitions. The dimensionless maximum velocity at the orifice $v_{o}^{*}(\max )$ is defined by $v_{o}^{*}=v_{o}(\max ) / U$, where $v_{o}(\max )$ is the maximum velocity at the aneurysm orifice. The mean velocity reduction $\bar{v}_{r}$ is defined as $\frac{\bar{v}^{n s}-\bar{v}^{s t}}{\bar{v}^{n s}} \times 100$, where $\bar{v}^{n s}$ and $\bar{v}^{s t}$ are the averaged nonstented velocity and the averaged stented velocity in the aneurysm dome respectively. The parameter $p_{a}^{*}$ is the dimensionless pressure in the aneurysm defined as $\frac{p_{a}-p_{\text {out }}}{p_{\text {in }}-p_{\text {out }}}$, where $p_{\text {in }}$ is the inlet pressure, $p_{\text {out }}$ is the outlet pressure. The parameter $p_{d}^{*}$ is the dimensionless pressure difference defined as $\frac{p_{a}^{\max }-p_{a}^{\min }}{p_{\text {in }}-p_{\text {out }}}$, where $p_{a}^{\text {max }}$ is the maximum pressure in the aneurysm and $p_{a}^{\text {min }}$ is the minimum pressure in the aneurysm. In Figs. 4 (a)-(i) we observe that the velocity component perpendicular to the main flow in the parent vessel (y velocity) is difficult to reduce in comparison with the velocity component parallel to the main flow ( $\mathrm{x}$ velocity). The y velocity is accelerated partly due to an 'orifice effect'. Table 2 shows also that this 'orifice effect' of the stent cannot be neglected so far as the pressure is concerned. When the flow reduction is incomplete, the orifice effect increases the y velocity. (see Fig. 4 (b), (e), (h), and (k)).

The experimental observation of Baráth et al. [11] using models with similar aspect ratio as LLa and Sa shows that model LLa has a better velocity reduction than model Sa when the stent porosity (metal free area/total unit area) ranges from $79 \%$ to $92.5 \%$ [11]. We analyze this reason here. In our numerical experiment we recognize this phenomenon in the large pore-size region (large porosity). Figures 5 (a)-(c) show that a ratio of the stent pore size to the aneurysm orifice size of 0.5 is the critical value, which makes the flow reduction effect in the model Sa smaller than that in the model LLa. In the region where this ratio is smaller than 0.5, model Sa has a better velocity reduction than model LLa. This crossover phenomenon is caused by the insufficient reduction of $\mathrm{x}$ velocity in model Sa when the pore size of the stent is large (large porosity) (see Fig. 5 (e)). This insufficient reduction is due to the small number of stent struts at the aneurysm orifice. When the ratio of the pore size to the aneurysm orifice is above 0.5 , there are only one or two struts at the orifice and the flow reduction shows the critical behavior. Usually for its flexibility the stent has large pore size compared to the aneurysm orifice size. Therefore it is very important to know the 
flow properties in this critical domain. On the other hand in the small pore size region (small porosity), model Sa can be occluded easier by the stent because of the large number of stent struts and the small velocity at the aneurysm orifice. This numerical analysis confirms well the experimental observation of Baráth et al. [11].
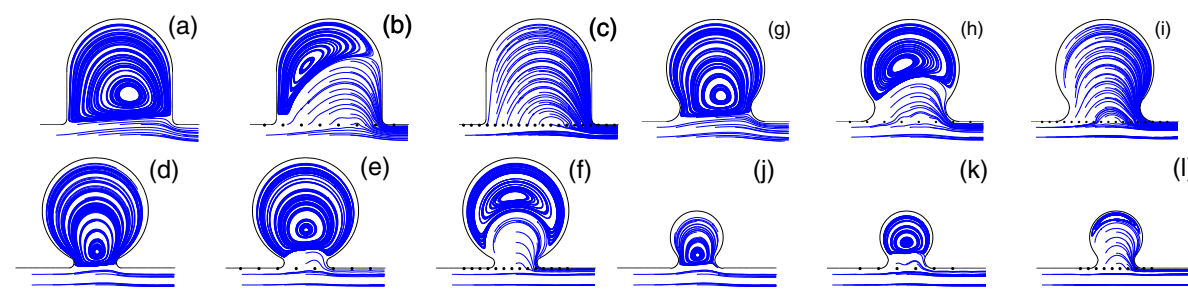

(k)

(I)

Fig. 3. Stream-line plots of the stent effect in aneurysms with the different orifice size (see aneurysm parameters and stent parameters in Table 2).
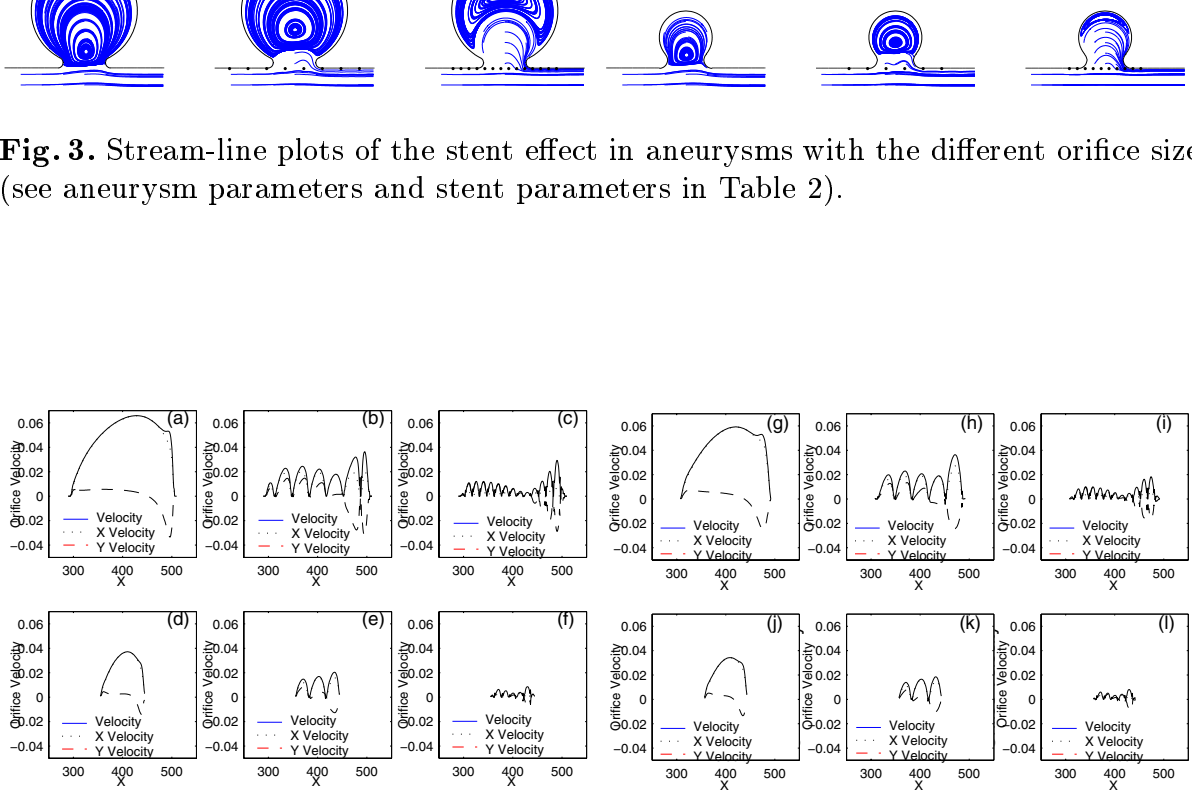

Fig. 4. The velocity profiles at the aneurysm orifice (a)-(l) of Fig. 3 (a)-(l) respectively.

\section{Conclusion}

Flow reduction mechanism in stented aneurysms has been discussed using the two-dimensional lattice Boltzmann approach. We showed that the stent prevents vortices driven directly by the parent vessel flow and that the aneurysm size plays an important role in the formation of the stented flow pattern. We need to consider the aneurysm size when we choose the suitable stent for flow reduction. The desirable stent should be designed to decrease the direct influence of the main flow. We need the sufficient number of stent struts at the aneurysm orifice, 

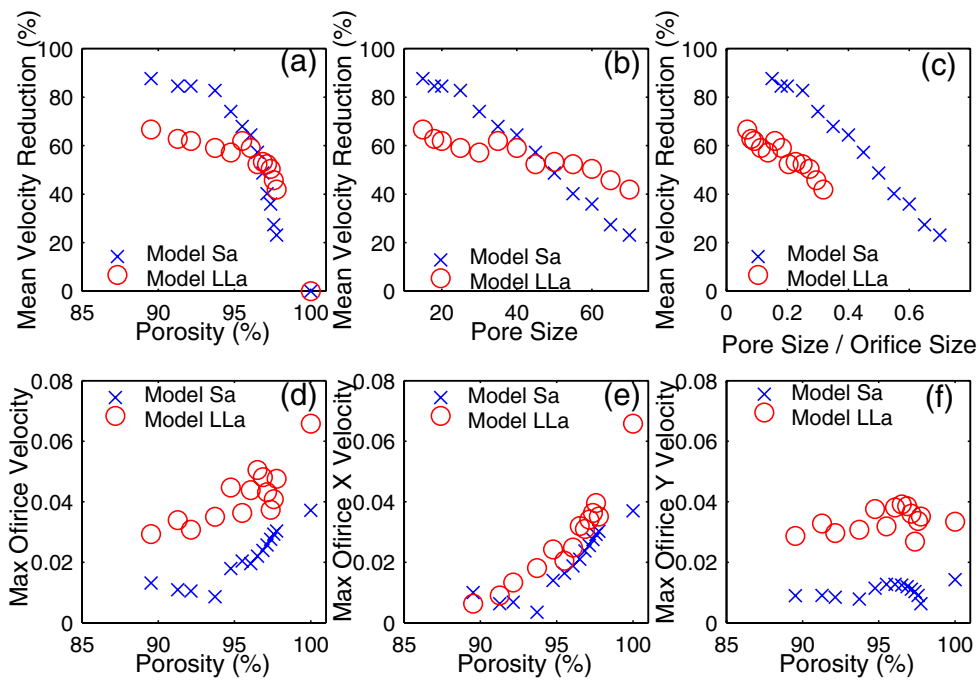

Fig. 5. The velocity reduction in the stented aneurysms. The mean velocity reductions in the aneurysm models LLa and Sa are shown in (a)-(c) as a function of porosity, pore size or the proportion of the pore size to the aneurysmal orifice size respectively. Porosity reduction is obtained by the simple pore size reduction. We plot the maximum velocity, maximum $x$ velocity and maximum y velocity at the aneurysm orifice as a function of the porosity in (d)-(f) respectively. The variation of the porosity is obtained by the simple pore-size reduction. The porosity $100 \%$ means the nonstented case.

Table 2. Parameters in fig. 3. In the column headings $v_{o}^{*}(\max )$ denotes the dimensionless maximum orifice velocity by the maximum velocity in the parent vessel, $\bar{v}_{r}$ is the mean velocity reduction, $p_{a}^{*}$ (whole) is the dimensionless pressure in the whole aneurysm and $p_{a}^{*}$ (upper) is that in the upper aneurysm, $p_{d}^{*}$ (upper) is the dimensionless pressure difference in the upper aneurysm.

\begin{tabular}{lcccccccr}
\hline Model & \multicolumn{2}{c}{ Aneurysm Stent $v_{o}^{*}(\max )$} & $\bar{v}_{r}(\%)$ & $p_{a}^{*}($ whole $)$ & $p_{a}^{*}$ (upper $)$ & $p_{d}^{*}($ upper $)$ & $v_{o}^{*} / p_{d}^{*}$ \\
\hline Fig. 3 (a) & LLa & - & 0.4910 & - & 1.0530 & 1.0350 & 0.00300 & 164 \\
Fig. 3 (b) & & Ls & 0.2709 & 61.90 & 1.0560 & 1.0410 & 0.00075 & 361 \\
Fig. 3 (c) & & Ss & 0.2187 & 66.67 & 1.0410 & 1.0350 & 0.00024 & 1154 \\
Fig. 3 (d) & Sa & - & 0.2769 & - & 1.0230 & 1.0380 & 0.00420 & 66 \\
Fig. 3 (e) & & Ls & 0.1522 & 67.88 & 1.0200 & 1.0410 & 0.00045 & 338 \\
Fig. 3 (f) & & Ss & 0.0642 & 87.64 & 0.9990 & 1.0320 & 0.00024 & 268 \\
Fig. 3 (g) & La & - & 0.4410 & - & 1.0470 & 1.0440 & 0.00450 & 98 \\
Fig. 3 (h) & & Ls & 0.2716 & 58.82 & 1.0350 & 1.0440 & 0.00090 & 302 \\
Fig. 3 (i) & & Ss & 0.1366 & 72.06 & 1.0080 & 1.0350 & 0.00015 & 911 \\
Fig. 3 (j) & SSa & - & 0.2552 & - & 0.0600 & 0.1020 & 0.00900 & 28 \\
Fig. 3 (k) & & Ls & 0.1373 & 56.76 & 0.9180 & 0.9840 & 0.00240 & 57 \\
Fig. 3 (l) & & Ss & 0.0597 & 79.44 & 0.8430 & 0.9780 & 0.00030 & 199 \\
\hline
\end{tabular}


therefore small pore size is preferable for the small-necked aneurysm, however it causes a kind of orifice effect and prevents the flow reduction in the large-necked aneurysm. These numerical observations can explain well the experimental results of Baráth et al. [11]. We need further discussion on the other important parameters, which defines the effective stent. In our future work, we would like to study the effect of the pressure on the elastic aneurysm wall and the effect of promoting the thrombosis by the stent implantation for deeper understanding of the vortex role in the aneurysm. In comparison with traditional methods such as finite element method, the lattice Boltzmann method is easy to handle due to the simple algorithm and shows promising capabilities to simulate complex flow such as non-Newtonian multi-phase flows [13,28] and moving elastic boundaries [29] without phenomenological models.

Acknowledgement. We acknowlege the great help of Krisztina Baráth, Francis Cassot, Jonas Lätt, and Alexandre Dupuis.

\section{References}

1. Vorp, D. A., Steinman, D. A., Ethier, C. R.: Computational Modeling of Arterial Biomechanics. IEEE Comput. Sci. Eng. 3(5) (2001) 51-64

2. Berger, S. A., Jou, L-D.: Flows in Stenotic Vessels. Annu. Rev. Fluid Mech. 32 (2000) 347-382

3. Wootten, D. M., Ku D. N.: Fluid Mechanics of Vascular Systems, Diseases, and Thrombosis. Annu. Rev. Biomed. Eng. 1 (1999) 299-329

4. Ku, D. N.: Blood Flow in Arteries. Annu. Rev. Fluid. Mech. 29 (1997) 399-434

5. Lieber, B. B., Stancampiano, A. P., Wakhloo, A. K.: Alteration of hemodynamics in aneurysm models by stenting: influence on stent porosity. Ann. Biomed. Eng. 25(3) (1997) 460-469

6. Marks, M. P., Dake, M. D., Steinberg, G. K., Norbash, A. M., Lane,B.: Stent Placement for Arterial and Venous Cerebrovascular Disease: Preliminary Clinical Experience. Radiology 191 (1994) 441-446

7. Wakhloo, A. K., Schellhammer, F., Vries, J. de., Haberstroh, J., Schumacher, M.: Self-expanding and ballon-expandable stents in the treatment of carotid aneurysms: an experimental study in a canie model, AJNR Am. J. Neuroradiol., 15 (1994) 493502

8. Aenis, M., Stancampiano, A. P., Wakhloo, A. K., Lieber, B. B.: Modeling of Flow in a Straight Stented and Nonstented side Wall Aneurysm Model. ASME Journal of Biomechanical Engineering 119 (1997) 206-212

9. Liou, T. M., Chang, W. C., Liao, C. C.: LDV measurements in lateral model aneurysms of various sizes. Exp. in Fluids. 23 (1997) 317-324

10. Yu, S. C. M., Zhao, J. B.: A steady flow analysis on the stented and non-stented sidewall aneurysm models. Med. Eng. Phys. 21 (1999) 133-141

11. Baráth, K., Cassot, F., Ohta, M., Fasel J. H. D., Rüfenacht, D. A.: Influence of stent properties on the alteration of hemodynamics in elastic cerebral aneurysm models. to be submitted

12. Succi, S.: The Lattice Boltzmann Equation for Fluid Dynamics and Beyond (Oxford Science Publications, 2001) 
13. Chen, S., Doolen, G. D.: Lattice Boltzmann method for fluid flows. Annu. Rev. Fluid Mech. 30 (1998) 329-364

14. Nichois W. W., O'Rourke, M. F.: McDonald's Blood Flows in Arteries, Lea and Febiger, Philadelphia-London (1974)

15. Perktold, K., Resch, M., Florian, H.: Pulsatile non-newtonian flow characteristics in a three-dimensional human carotid bifurcation model. ASME Journal of Biomechanical engineering 113 (1991) 464-475

16. Higauera, F., Succi, S., Benzi, R.: Lattice gas dynamics with enhanced collisions. Europhys. Lett. 9 (1989) 345-49

17. Qian, Y. H., d'Humiéres, D., Lallemand, P.: Lattice BGK models for Navier-Stokes equation. Europhys. Lett. 17 (1992) 479-484

18. Chen, H., Chen, S., Matthaeus, W.: Recovery of the Navier-Stokes equations using a lattice-gas Boltzmann method. Phys. Rev. A. 45 (1992) R5339-R5342

19. Bhatnagar, P.L., Gross E.P., Krook M.: A model for collision processes in gases. I: small amplitude processes in charged and neutral one-component system. Phys. Rev. 94 (1995) 511-525

20. Koelman, J.M.V.A.: A simple lattice Boltzmann scheme for Navier-Stokes fluid flow. Europhys. Lett. 15 (1991) 603-607

21. He, X., Zou, Q., Luo, L. S., Dembo, M.: Analytic solutions of simple flow and analysis of non-slip boundary conditions for the lattice Boltzmann BGK model. J. of Stat. Phys. 87 (1997) 115-136

22. Dupuis, A.: From a lattice Boltzmann model to a parallel and reusable implementation of a virtual river, Doctoral thesis, University of Geneva (2002). http://cui.unige.ch/spc/PhDs/aDupuisPhD/phd.html

23. Ziegler, D. P.: Boundary conditions for lattice Boltzmann simulations. J. Sta. Phys. 71 (1993) 1171-1177

24. Skordos, P. A.: Initial and boundary conditions for the lattice Boltzmann method. Phys. Rev. E. 46 (1993) 4823-4842

25. Löw, M., Perktold, K., Rauning, R.: Hemodynamics in Rigid and Distensible Saccular Aneurysms: A Numerical Study of Pulsatile Flow Characteristics. Biorheology 30 (1993) 287-298

26. Perktold, K., Kenner, T., Hilbert, D., Spork, B., Florian, H.: Numerical Blood Flow Analysis: Arterial Bifurcation With a Saccular Aneurysm. Basic Res. in Cardiol. 83 (1988) 24-31

27. Perktold, K., Peter, R., Resch, M.: Pulsatile Non-Newtonian Blood Flow Simulation Through a Bifurcation With an Aneurysm. Biorheology 26 (1989) 1011-1030

28. Giraud, L., D'Humiéres, D., Lallemand, P.: A lattice Boltzmann model for Jeffreys viscoelastic fluid, Europhys. Lett., 46 (1998) 625-630

29. Chopard, B., Marconi, S.: Lattice Boltzmann Solid Particles in a Lattice Boltzmann Fluid. J. Stat. Phys. 107 (2002) 23-37 